\title{
MOG encephalomyelitis: international recommendations on diagnosis and antibody testing
}

\author{
S. Jarius ${ }^{1 *}$, F. Paul ${ }^{2,3,4}$, O. Aktas ${ }^{5}$, N. Asgari ${ }^{6}$, R. C. Dale ${ }^{7}$, J. de Seze ${ }^{8}$, D. Franciotta ${ }^{9}$, K. Fujihara ${ }^{10}$, A. Jacob ${ }^{11}$, \\ H. J. Kim ${ }^{12}$, I. Kleiter ${ }^{13}$, T. Kümpfel ${ }^{14}$, M. Levy ${ }^{15}$, J. Palace ${ }^{16}$, K. Ruprecht $^{4}$, A. Saiz ${ }^{17}$, C. Trebst ${ }^{18}$, B. G. Weinshenker ${ }^{19}$ \\ and B. Wildemann ${ }^{1 *}$
}

\begin{abstract}
Over the past few years, new-generation cell-based assays have demonstrated a robust association of autoantibodies to full-length human myelin oligodendrocyte glycoprotein (MOG-lgG) with (mostly recurrent) optic neuritis, myelitis and brainstem encephalitis, as well as with acute disseminated encephalomyelitis (ADEM)-like presentations. Most experts now consider MOG-IgG-associated encephalomyelitis (MOG-EM) a disease entity in its own right, immunopathogenetically distinct from both classic multiple sclerosis (MS) and aquaporin-4 (AQP4)-IgG-positive neuromyelitis optica spectrum disorders (NMOSD). Owing to a substantial overlap in clinicoradiological presentation, MOG-EM was often unwittingly misdiagnosed as MS in the past. Accordingly, increasing numbers of patients with suspected or established MS are currently being tested for MOG-IgG. However, screening of large unselected cohorts for rare biomarkers can significantly reduce the positive predictive value of a test. To lessen the hazard of overdiagnosing MOG-EM, which may lead to inappropriate treatment, more selective criteria for MOG-lgG testing are urgently needed. In this paper, we propose indications for MOG-lgG testing based on expert consensus. In addition, we give a list of conditions atypical for MOG-EM ("red flags") that should prompt physicians to challenge a positive MOG-IgG test result. Finally, we provide recommendations regarding assay methodology, specimen sampling and data interpretation.
\end{abstract}

Keywords: Myelin oligodendrocyte glycoprotein (MOG) antibodies, Consensus recommendations, Diagnosis, Antibody testing, Multiple sclerosis (MS), Neuromyelitis optica spectrum disorders (NMOSD), Optic neuritis (ON), Myelitis

\section{Background}

Over the past few years, the role of immunoglobulin G serum antibodies to myelin oligodendrocyte glycoprotein (MOG-IgG) in patients with inflammatory CNS demyelination has been revisited. While antibodies to MOG were originally thought to be involved in multiple sclerosis (MS), based on results from enzyme-linked immunosorbent assays employing linearized or denatured MOG peptides as antigen, more recent studies using new-generation cellbased assays have demonstrated a robust association of antibodies to full-length, conformationally intact human MOG protein with (mostly recurrent) optic neuritis $(\mathrm{ON})$,

\footnotetext{
* Correspondence: sven.jarius@med.uni-heidelberg.de;

brigitte.wildemann@med.uni-heidelberg.de

${ }^{1}$ Molecular Neuroimmunology Group, Department of Neurology, University Hospital Heidelberg, Im Neuenheimer Feld 350, 69120 Heidelberg, Germany Full list of author information is available at the end of the article
}

myelitis and brainstem encephalitis, as well as with acute disseminated encephalomyelitis (ADEM)-like presentations, rather than with classic MS [1-11].

Based on evidence from (a) immunological studies suggesting a direct pathogenic impact of MOG-IgG, (b) neuropathological studies demonstrating discrete histopathological features, (c) serological studies reporting a lack of aquaporin-4 (AQP4)-IgG in almost all MOGIgG-positive patients, and (d) cohort studies suggesting differences in clinical and paraclinical presentation, treatment response and prognosis, MOG-IgG is now considered to denote a disease entity in its own right, distinct from classic MS and from AQP4-IgG-positive neuromyelitis optica spectrum disorders (NMOSD), which is now often referred to as MOG-IgG-associated encephalomyelitis (MOG-EM) [11-13].

(c) The Author(s). 2018 Open Access This article is distributed under the terms of the Creative Commons Attribution 4.0 International License (http://creativecommons.org/licenses/by/4.0/), which permits unrestricted use, distribution, and 
Importantly, however, MOG-EM and MS show a relevant phenotypic, i.e., clinical as well as radiological, overlap [3, 14]: like MS, MOG-EM follows a relapsing course in most cases [3, 6], at least in adults, and 33 and $15 \%$ of adult patients with MOG-EM meet McDonald's and Barkhof's criteria for MS, respectively, at least once over the course of disease [3, 14]. Accordingly, many patients with MOG-EM were falsely classified as having MS in the past $[3,4]$. However, such misclassification has potential therapeutic implications: (a) similar to what has been observed in AQP4-IgG-positive NMOSD, some drugs approved for MS might be ineffective or even harmful in MOG-EM owing to differences in immunopathogenesis [3, 4, 15-17]; (b) MOG-EM is associated with a high risk of flare-ups after cessation of steroid treatment for acute attacks and may thus require close monitoring and careful steroid tapering [3, 18-22]; and (c) patients positive for MOG-IgG might be particularly responsive to antibody-depleting treatments for acute attacks such as plasma exchange or immunoadsorption [3, 4, 9, 14, 23, 24], to $B$ cell-targeted long-term therapies such as rituximab, to treatment with intravenous immunoglobulins (IVIG) (especially in children [25]), and to immunosuppressive treatments $[3,6,14,25,26]$. Therefore, increasing numbers of patients with suspected or established MS are currently being screened for MOG-IgG.

However, screening of large unselected populations for rare biomarkers generally decreases the positive predictive value of diagnostic tests by increasing the rate of falsepositive results $[27,28]$. Even if assays with high specificity $(\geq 99 \%)$ are used, true-positive (TP) results can easily be outnumbered by false-positive (FP) results if the prevalence of a marker is low and the number of samples tested is high. This also applies to MOG-IgG testing. Based on a hypothetical prevalence of $1 \%$ genuinely MOG-IgGpositive cases among all patients currently diagnosed with MS, testing of 100,000 patients with an almost flawless, $99 \%$ specific and $100 \%$ sensitive assay would result in an unacceptable ratio of $990 \mathrm{FP}$ results to $1000 \mathrm{TP}$ results. Therefore, unselected screening of all patients with suspected or established MS for MOG-IgG should be discouraged and more specific criteria for MOG-IgG testing are urgently needed.

In this paper, we propose for the first time indications for MOG-IgG testing based on expert consensus. In addition, we give a list of conditions considered atypical for MOGEM ("red flags") that should prompt physicians to challenge the validity of a positive MOG-IgG test result. Finally, we provide recommendations regarding assay methodology, specimen sampling, and data interpretation.

\section{Methods}

PubMed was searched for articles published between February 2007 and February 2017 using the following search term: ("myelin oligodendrocyte glycoprotein" OR MOG) AND (antibody OR antibodies OR IgG). All articles identified by this means were reviewed by a core group of physicians (S.J., B.W., F.P., K.R.) for clinical and paraclinical findings that have been frequently reported in association with MOG-IgG seropositivity in patients with CNS demyelination and which, therefore, may justify MOG-IgG testing, as well as for potential "red flags", i.e., conditions that are typically found in inflammatory disorders of the CNS but have been reported to be absent or very rare in MOG-IgG-positive patients and thus may indicate diagnoses other than MOG-EM. Based on core group consensus, a first set of recommendations was formulated and then circulated to a broader panel of experts in the field from Australia, Denmark, France, Germany, Italy, Japan, South Korea, Spain, UK, and the USA for discussion and refinement. Panel members were invited by the core group based on eminence and previous contributions to the field. Based on several rounds of core group-led peer-to-peer discussions of the individual recommendations with all individual members of the panel, a final set of evidence- as well as eminence-based recommendations was drawn up to which all members gave their approval. All recommendations given here should be considered as expert consensus.

\section{Recommendations on MOG-IgG testing}

In Table 1, we propose indications for MOG-IgG testing based on clinical and paraclinical findings that are typical of MOG-EM and/or atypical for MS and were considered by the panel members to be associated with pre-test odds high enough to justify MOG-IgG testing or that demand MOG-IgG testing because of potentially significant therapeutic consequences of a positive test result according to expert consensus. These recommendations apply to all patients with suspected CNS demyelination of putative autoimmune etiology and an either monophasic or relapsing disease course. Given the very low pre-test probability [29], we recommend against general MOG-IgG testing in patients with a progressive disease course. In Table 2, we give a number of case vignettes of patients considered to be at high risk of MOG-EM to illustrate the broad spectrum of symptoms associated with that syndrome and the practical feasibility and relevance of the proposed criteria. In Table 3, we give a number of recommendations regarding assay selection, specimen sampling, and data interpretation. Finally, Table 4 lists conditions ("red flags") that we believe are atypical for MOG-EM and should thus prompt physicians to challenge a positive MOG-IgG test result and seek a better explanation for the patients' clinical and paraclinical findings.

In practice, many patients diagnosed with AQP4-IgGnegative NMOSD according to the IPND 2015 criteria 
Table 1 Recommended indications for MOG-lgG testing in patients presenting with acute CNS demyelination of putative autoimmune etiology

1. Monophasic or relapsing acute optic neuritis, myelitis, brainstem encephalitis, encephalitis, or any combination thereof, and

2. radiological or, only in patients with a history of optic neuritis, electrophysiological (VEP) findings compatible with CNS demyelination, and

3. at least one of the following findings:

MRI

a. Longitudinally extensive spinal cord lesion ( $\geq 3 \mathrm{VS}$, contiguous) on MRI (so-called LETM) a,b

b. Longitudinally extensive spinal cord atrophy ( $\geq 3$ VS, contiguous) on MRI in patients with a history compatible with acute myelitis ${ }^{\mathrm{a}}$ c. Conus medullaris lesions, especially if present at onset ${ }^{\mathrm{C}}$

d. Longitudinally extensive optic nerve lesion (e.g., $>1 / 2$ of the length of the pre-chiasmal optic nerve, $\mathrm{T} 2$ or $\mathrm{T} 1 / \mathrm{Gd})^{\mathrm{d}}$

e. Perioptic Gd enhancement during acute $\mathrm{ON}^{\mathrm{e}}$

f. Normal supratentorial MRI in patients with acute ON, myelitis and/or brainstem encephalitis

g. Brain MRI abnormal but no lesion adjacent to a lateral ventricle that is ovoid/round or associated with an inferior temporal lobe lesion and no Dawson's finger-type or juxtacortical U fiber lesion (Matthews-Jurynczyk criteria')

h. Large, confluent T2 brain lesions suggestive of ADEM

Fundoscopy

i. Prominent papilledema/papillitis/optic disc swelling during acute ON

j. Neutrophilic CSF pleocytosis ${ }^{9}$ or CSF WCC $>50 /\left.\mu\right|^{\text {h }}$

k. No CSF-restricted OCB as detected by IEF at first or any follow-up examination' (applies to continental European patients only)

Histopathology

I. Primary demyelination with intralesional complement and lgG deposits

m. Previous diagnosis of "pattern II MS" $\mathrm{j}$

Clinical findings

n. Simultaneous bilateral acute ON

o. Unusually high ON frequency or disease mainly characterized by recurrent ON

p. Particularly severe visual deficit/blindness in one or both eyes during or after acute ON

q. Particularly severe or frequent episodes of acute myelitis or brainstem encephalitis

r. Permanent sphincter and/or erectile disorder after myelitis

s. Patients diagnosed with "ADEM", "recurrent ADEM", "multiphasic ADEM" or "ADEM-ON"

t. Acute respiratory insufficiency, disturbance of consciousness, behavioral changes, or epileptic seizures (radiological signs of demyelination required)

u. Disease started within 4 days to $\sim 4$ weeks after vaccination

v. Otherwise unexplained intractable nausea and vomiting or intractable hiccups (compatible with area postrema syndrome) ${ }^{a}$

w. Co-existing teratoma or NMDAR encephalitis (low evidence ${ }^{k}$ )

Treatment response

x. Frequent flare-ups after IVMP, or steroid-dependent symptoms' (including CRION)

y. Clear increase in relapse rate following treatment with IFN-beta or natalizumab in patients diagnosed with MS (low evidence)

Note that these recommendations are primarily intended for use in adults and adolescents. Indications for MOG-IgG testing in young children need not to be as rigorous as in adults, since MOG-EM is thought to be significantly more frequent among young children with acquired demyelinating disease (up to $70 \%$; frequency declining with age) than among their adult counterparts ( $\leq 1 \%$ in Western countries; probably $\leq 5 \%$ in Japan and other Asian countries because of lower MS prevalence), which reduces the risks attached to antibody screening outlined in the Introduction

Abbreviations: $A D E M$ acute disseminated encephalomyelitis, $A D E M-O N$ ADEM with recurrent $O N, A Q P 4$ aquaporin-4, CNS central nervous system, CRION chronic relapsing inflammatory optic neuropathy, CSF cerebrospinal fluid, EM encephalomyelitis, Gd gadolinium, IA immunoadsorption, IgG immunoglobulin G, IVMP intravenous methylprednisolone, LE left eye, LETM longitudinally extensive transverse myelitis, MOG myelin oligodendrocyte glycoprotein, MRI magnetic resonance imaging, MS multiple sclerosis, NMDAR N-methyl-D-aspartate receptor, NMO neuromyelitis optica, OCB oligoclonal lgG bands, ON optic neuritis, $P E X$ plasma exchange, RE right eye, RRMS relapsing-remitting MS, VEP visual evoked potentials, VS vertebral segments, WCC white cell count

af costs play a role and disease is stable: test AQP4-lgG first, since more frequent in that condition than MOG-IgG. If disease is active, requiring fast decision-making, or if costs play no role: test AQP4-IgG and MOG-IgG in parallel

${ }^{b}$ LETM is common both in MOG-EM and in AQP4-NMOSD, but rarely if ever occurs in MS; as a caveat, however, non-contiguous lesions may mimic LETM in some patients with MS. N.B.: Short lesions do not per se exclude MOG-EM. MRI shows short lesions at least once over the disease course in around 44-52\% of all MOG-EM patients [3,39] and around 15\% of all AQP4-NMOSD patients [40]. Lesion length may also depend on MRI timing issues, with shorter lesions detected when the MRI was performed early in the evolution of acute myelitis or in clinical remission. Both axial and sagittal plane images should be used to judge lesion extent. LETM has also been shown to be frequently present at disease onset in MOG-IgG-positive children with manifestations other than isolated ON (32/40 or $80 \%$ of all examined cases) [41]

'Present in $6 / 8$ patients in [7] (at onset); $4 / 6$ in [8]; 4/11 in [35]; 3/12 in [42]; 5/26 (not all had lumbar MRI) in [3]; and in 13/40 pediatric patients (at onset) with manifestations other than isolated ON [41]

${ }^{\mathrm{d}}$ Ramanathan et al. (2015) reported a median optic nerve lesion length of $23.1 \mathrm{~mm}$ (IQR 18-33) in MOG-lgG-related ON (N=19); this compares to a median lesion length of $9.9 \mathrm{~mm}$ (IQR 6.6-19.8; $\mathrm{N}=13$ ) in MS-related ON observed in the same study [43] and of $10.5 \mathrm{~mm}$ in a second, independent study ( $\mathrm{N}=26$ ) [44]. Recent data suggest that also involvement (T2, T1/Gd or optic nerve swelling) of > 6/12 optic nerve segments (anterior orbital RE/LE, posterior orbital RE/LE, canalicular RE/LE, intracranial RE/LE, chiasm right/left half, optic tract right/left side) may be associated with increased pre-test odds for MOG-lgG (observed in 6/19 [32\%] MOG-lgGpositive ON patients but in none of 13 [0\%] MS-ON patients) [43]. Longitudinal extensive lesions involving at least 4 of 5 segments (anterior intraorbital segment, posterior intraorbital segment, canalicular, intracranial, chiasmal) were also noted in $>=50 \%$ of MOG-IgG-positive patients in [45]. By contrast, lesions in MS-related ON extended only over 1 (70\%) or $2(30 \%)$ of 9 segments (intraorbital RE/LE, canalicular RE/LE, intracranial RE/LE, chiasmal, optic tract right/left side) in [44], and a mean extension of just 2.2/10 segments (orbital RE/LE, canalicular RE/LE, intracranial RE/LE, chiasma right/left half, optic tract right/left side) was observed in MS$\mathrm{ON}$ in [46]. Longitudinal extensive lesions ranging over more than the half of the distance between the optic nerve head and the chiasm were also reported in $3 /$ 3 patients in [47] and in 6/10 (60\%) in [3]. Finally, 9/10 MOG-ON Han patients (90\%) showed involvement of all three segments of the pre-chiasmal optic nerve (intraorbital, canalicular, intracranial) in [48], in 6 of whom chiasmal and/or optic tract involvement was noted in addition

eObserved in $11 / 28$ patients during acute ON in [3], in 6/18 in [49], and in 6/8 in [48], but not usually in MS. Perioptic T2 hyperintensity alone does not count 
Table 1 (Continued)

${ }^{f}$ Positive in $\geq 90 \%$ of RRMS patients $[37,36,50]$. By contrast, ovoid/round lesions adjacent to a lateral ventricle, lesions adjacent to a lateral ventricle in association with a temporal lobe lesion, and Dawson's finger-type lesions were absent in 21/21 (100\%) MOG-lgG-positive patients in a mixed adult $(n=15)$ and pediatric $(n=6)$ cohort [36, 37] and juxtacortical U fiber lesions in 20/21 (95.2\%). Recently, a lack of Dawson's finger-type lesions in MOG-lgG-positive patients has been confirmed in an exclusively pediatric cohort (absent in 68/69 [98.6\%]; the only patient positive for Dawson's finger lesions had typical MS and was negative for MOG-lgG at re-testing); $U$ fiber lesions were absent in 65/69 (94.2\%) MOG-IgG-positive pediatric patients in the same study [41]

${ }^{9}$ Present at least once in $64 \%$ of patients with pleocytosis [3] (median $22 \%$ of all white cells; range 3-69\%) but typically absent in MS. N.B.: Neutrophilic pleocytosis is also frequently found in AQP4-IgG-positive NMOSD [51]

hObserved in 43\% (14/36) of MOG-IgG-positive patients with pleocytosis (peak values) [3], but only rarely in patients with MS ( $\leq 2 \%$ according to [52]; $1 / 71$ patient $\geq 15$ years of age [range 15-29] in [53])

i Oligoclonal bands (OCB) have been reported in up to $98 \%$ of patients with MS in central and Northern Europe [53] but only in around 12-13\% of patients with MOG-EM in two recent Central European studies [3,54]; of note, many MOG-EM patients previously falsely diagnosed with MS were atypical in that they had no OCB in a recent multicenter study [3]. As a caveat, it should be noted that positive OCB do NOT exclude MOG-EM [3] and that the frequency of OCB in MS may be lower in Asian patients (e.g., 40-80\% in Japan) as well as in some regions in Europe such as Sardinia (84\% in a recent study [55]), possibly depending on genetic factors. "No CSF-restricted OCB" refers to the presence of OCB patterns 1 (no OCB), 4 (mirror pattern without additional lgG bands present exclusively in the CSF), or 5 (monoclonal lgG band present both in the CSF and in the serum) [56]

jSome patients diagnosed with "pattern II MS" lesions, which are characterized by lgG and complement deposits, were shown by independent groups to have in fact MOG-EM, suggesting that the current histopathological criteria may not be sufficiently specific to distinguish between MS and MOG-EM [24, 57, 58] ${ }^{k}$ Patients with teratoma and positive MOG-IgG serostatus have been identified in two cohorts so far (2/74; 3\%) [3, 4, 59]; expression of CNPase, an oligodendrocyte marker, has been described in mature teratomas, and oligodendrogliomas may arise in mature teratomas. Additional testing for NMDAR antibodies is highly recommended in patients with teratoma and neurological symptoms [60]. Recent, though preliminary, reports suggest that MOG-EM and NMDAR encephalitis may occasionally co-exist [61] Re-occurrence of symptoms after tapering of oral steroids $[3,18,20,22,62]$

[30] will meet also the criteria for MOG-IgG testing given in Table 1 and should thus be tested. However, MOG-IgG testing should not be restricted to patients with AQP4-IgG-negative NMOSD. While this approach seems to offer simplicity, it would be inappropriate for several reasons: (1) The IPND criteria for AQP4-IgGnegative NMOSD demand dissemination in space, which would prevent testing of many patients with syndromes compatible with MOG-EM (e.g., patients with isolated longitudinally extensive transverse myelitis [LETM], isolated bilateral $\mathrm{ON}$, or isolated brainstem encephalitis). (2) They include magnetic resonance imaging (MRI) criteria that are based on lesion distribution patterns observed in AQP4-IgG-positive NMOSD, some of which reflect areas of high AQP4 expression; however, AQP4 is not the target antigen in MOG-EM. Accordingly, lesion distribution may differ between NMOSD and MOGEM and some MOG-EM patients do not satisfy these criteria (e.g., patients with recurrent bilateral non-longitudinal $\mathrm{ON}$ without chiasm involvement plus non-NMOSD-typical brain lesions, those with severe and recurrent non-longitudinally extensive myelitis, and those with ADEM-like presentation with severe brain and brainstem involvement but no area postrema lesion). (3) Such a recommendation would imply testing of all patients for AQP4-IgG before they were tested for MOG-IgG, which might unnecessarily delay diagnosis and treatment. (4) The criteria for AQP4-IgG-negative NMOSD require exclusion of other diagnoses; this would constitute a logical repugnancy, since a

Table 2 Case vignettes of patients at risk of MOG-lgG seropositivity (examples)

Example 1: 35-year-old woman presenting with bilateral acute ON. Develops transient blindness; fundoscopy shows papilledema; lumbar puncture reveals lymphomonocytic pleocytosis with 10\% neutrophils and negative OCBs; brain MRI shows perioptic Gd enhancement but is otherwise normal; flaring up of symptoms after tapering of oral steroids; later recurrent ON attacks, stabilization with rituximab.

Example 2: 40-year-old woman with two attacks of acute, OCB-negative myelitis. Spine MRI shows an isolated short spinal cord lesion at first attack and a longitudinally extensive spinal cord lesion at relapse; brain MRI abnormal but no Dawson's finger-type lesion, no juxtacortical U fibre lesion, and no lesion adjacent to a lateral ventricle that is ovoid or associated with an inferior temporal lobe lesion [36, 37, 50]; flaring up of myelitis symptoms after discontinuation of intravenous steroid treatment, good response to PEX.

Example 3: Young man with a previous diagnosis of "OCB-negative RRMS". Predominantly ON and myelitis attacks; conus lesion with severe erectile and sphincter disturbance after first myelitis; longitudinally extensive optic nerve lesion with involvement of the optic chiasm; increase in relapse rate under treatment with interferon-beta but stabilization with rituximab.

Example 4: 42-year-old woman presenting with incomplete, painful tetraparesis. Previous diagnosis of RRMS with positive OCB; spinal cord MRI reveals a contiguous lesion extending from C3 to T1; negative serology for AQP4-lgG.

Example 5: ADEM-like presentation with large white matter lesions and disturbance of consciousness, brainstem lesions, and involvement of the entire spinal cord in a 25-year-old woman; onset 3 weeks after vaccination.

Example 6: Simultaneous unilateral ON and LETM extending into the brainstem in a 39-year-old man. CSF pleocytosis (90 white cells/ $/$ l) with 5\% neutrophils; no CSF-restricted OCB; negative AQP4-IgG serostatus.

Example 7: Young woman presenting with recurrent and steroid-dependent isolated ON, previously classified as CRION; normal brain MRI.

Example 8: Young man with acute encephalitis and seizures. MRI reveals large cortical/subcortical white matter lesions not involving the inferior temporal lobe; good response to steroids; negative for typical viral and autoimmune causes of encephalitis.

Abbreviations: ADEM acute disseminated encephalomyelitis, AQP4 aquaporin-4, CRION chronic relapsing inflammatory optic neuropathy, CSF cerebrospinal fluid, $E M$ encephalomyelitis, Gd gadolinium, IgG immunoglobulin G, LETM longitudinally extensive transverse myelitis, MOG myelin oligodendrocyte glycoprotein, MRI magnetic resonance tomography, MS multiple sclerosis, ON optic neuritis, RRMS relapsing-remitting MS 
Table 3 Recommendations on methodology, test parameters, specimen sampling and data interpretation

\begin{abstract}
Assay types
Cell-based assays (IFT/FACS): Recommended (current gold standard); must employ full-length human MOG as target antigen; use of Fc-specific (or lgG1specific [63]) secondary antibodies highly recommended to avoid cross-reactivity with (specifically or non-specifically co-binding) IgM and IgA antibodies $[11,63]$

Immunohistochemistry: Currently not recommended (less sensitive than cell-based assays, limited data available on specificity [11, 64], sensitivity depends on tissue donor species [64]); if used, Fc-specific secondary antibodies adsorbed against tissue donor lgG required in order to avoid cross-reactivity with IgM and IgA or with tissue-bound donor lgG

Peptide-based ELISA, Western blot: Insufficiently specific, obsolete
\end{abstract}

Biomaterial

Serum: Recommended (specimen of choice); shipment at $4^{\circ} \mathrm{C}$ or on dry ice advisable if samples do no arrive within $1-2$ days

Cerebrospinal fluid: Not usually required, since MOG-IgG is produced mostly extrathecally, resulting in lower CSF than serum titers [2]; potentially helpful in rare, selected cases (e.g., strong background due to co-existing high-titer non-MOG serum antibodies); shipment at $4{ }^{\circ} \mathrm{C}$ or on dry ice advisable

Immunoglobulin classes

Testing for MOG-IgG: Recommended

Testing for MOG-IgM and/Or MOG-IgA: Currently not recommended; additional MOG-IgM and MOG-IgA antibodies have been described in some MOG-IgG-positive patients [1, 2]; the clinical relevance of isolated MOG-IgM or -lgA results is unknown; testing for antibodies of the IgM class requires removal of total IgG from the sample to avoid both false-negative (due to high-affinity lgG displacing IgM) and false-positive (due to $\lg M$ anti-lgG $G_{F c}$ rheumatoid factors) results [65]

\title{
Data reporting
}

Immunoglobulin class detected, assay type, antigenic substrate and biomaterial used, titer/concentration/units, assay-specific cut-offs and performing laboratory should all be documented (e.g., "Serum MOG-IgG 1:1280 [cut-off $\geq 1: 160^{a}$; assay: live CBA, Innsbruck lab; antigen: full-length human $\left.\mathrm{MOG}]^{\prime \prime}\right)$

\section{Data interpretation}

As with all laboratory tests, positive test results should always be interpreted in the context of the patient's overall presentation; if "red flags" as defined in Table 4 are present, re-testing of the positive serum sample (or, if not anymore available, at least testing of a follow-up serum sample) is recommended; to reduce the potential risk of reproducing false-positive results due to issues inherent to the very method employed, use of a second (and, in the case of discrepant results, third) methodologically different cell-based assay is advisable; if in doubt, seek expert advice from a specialized center

\section{Timing issues}

MOG-IgG serum concentrations depend on disease activity (with higher median concentrations during acute attacks than during remission) and treatment status (with lower concentrations while on immunosuppression) and may transiently vanish after plasma exchange [3]; if MOG-lgG is negative but MOG-EM still suspected, re-testing during acute attacks, during treatment-free intervals, or 1-3 months after plasma exchange (or $I V I G^{b}$ ) is recommended - N.B.: Some cases of monophasic MOG-positive EM/ADEM in adult patients have been described in which MOG-IgG disappeared permanently following clinical recovery [2-4, 33-35]

Abbreviations: ADEM acute disseminated EM, CBA cell-based assay, CSF cerebrospinal fluid, ELISA enzyme-linked immunosorbent assay, EM encephalomyelitis, FACS fluorescence-activated cell sorting, IgG/A/M immunoglobulin G/A/M, IFT indirect fluorescence test, IVIG intravenous immunoglobulins, MOG myelin oligodendrocyte glycoprotein, PEX plasma exchange

${ }^{2}$ Note that the cut-off given here is an example only; actual cut-off values are assay-dependent.

${ }^{b}$ Generally, pretreatment with IVIG is liable to cause false negative and false positive results in antibody assays [66-68]; whether any of the tests currently used for detecting MOG-IgG are affected by IVIG pretreatment has not been investigated so far.

negative test for MOG-IgG would be a prerequisite for MOG-IgG testing. (5) Finally, but less importantly, using NMOSD criteria for diagnosing MOG-EM would, in addition to resulting in a substantial loss in sensitivity and specificity, also be confusing to nonexperts, given that AQP4-IgG-positive NMOSD and MOG-EM are distinct diseases with different target antigens (AQP4 vs. MOG), pathophysiology (astrocytopathy vs. primary demyelination), and clinical spectra.

Alternatively, should we restrict MOG-IgG testing to patients with AQP4-IgG-negative NMO according to Wingerchuk's 2006 criteria [31]? This would again result in a substantial loss of patients at high risk of MOG$\mathrm{EM}$, since those criteria require a history of both $\mathrm{ON}$ and myelitis and would thus be inappropriate. Of note,
MOG-IgG testing in patients with seronegative NMO according to the 2006 criteria is already covered by our recommendation to test all patients with LETM for MOG-IgG (see Table 1), since the 2006 criteria strictly require a history of LETM in patients negative for AQP4-IgG.

Instead, we propose to base the indication for MOG-IgG testing in patients with suspected CNS demyelination on the presence of specific clinical and paraclinical findings that are considered typical for MOG-EM and/or atypical for conventional MS (see Table 1).

During the consensus-finding process, concerns were raised regarding inclusion of the following treatment-related indications for MOG-IgG testing in Table 1: 
a. Particularly good response to antibody-depleting therapies (plasma exchange [PEX], immunoadsorption [IA])

b. Particularly good response to B cell-depleting therapies (rituximab, ocrelizumab, ofatumumab) but relapse immediately after re-occurrence of B cells

It was argued by some members of the panel that good responses to PEX, IA, or B cell depletion have also been observed in conventional MS. However, consensus was achieved that if present in addition to any of the indications listed in Table 1 , good response to antibody or B cell-depleting treatments or IVIG further increases the pre-test likelihood of MOG-EM and thus supports the decision to test for MOG-IgG.

Taking into account that MOG-IgG serum concentrations depend on disease activity (with higher concentrations during acute attacks) and treatment status (with lower concentrations while on immunosuppression) as well as on assay sensitivity, we recommend re-testing patients during acute attacks or during treatment-free intervals and/or in a second cell-based assay if MOGIgG was negative at first examination but MOG-EM is still suspected based on the list of indications given in Table 1 [3].

Only sparse data are available on the usefulness of regular monitoring of antibody titers in individual patients known to be positive for MOG-IgG. Median MOG-IgG titers have indeed been shown to be significantly higher during relapse than during remission [2], making regular MOG-IgG testing a potentially promising method for predicting attacks and monitoring treatment efficacy. However, there are several limitations: While titers $>1: 2560$ were found only during acute attacks in a recent study using a live cell-based assay [2], some patients still had relatively low titers during acute attacks and others had relatively high titers during remission, suggesting that additional factors such as blood-CSF barrier damage, $\mathrm{T}$ cell activation, antibody affinity, or complement-activating activity may be involved, with no general cut-off value for relapse induction [2]. In addition, treatment effects could

Table 4 "Red flags": conditions that should prompt physicians to challenge a positive test result (consider re-testing the patient, ideally using an alternative, i.e., methodologically different cell-based assay; in case of doubt, consider seeking expert advice from a specialized center)

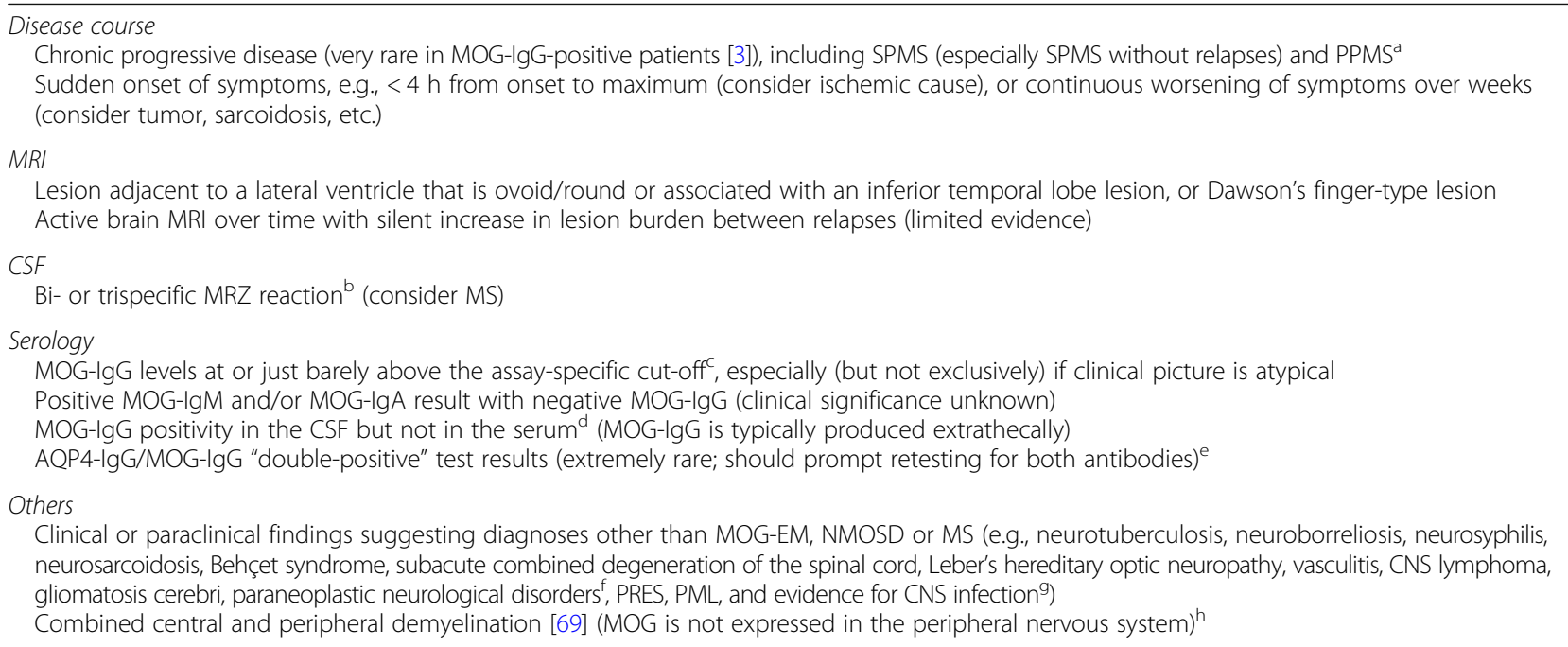

Abbreviations: AQP4 aquaporin-4, CNS central nervous system, CSF cerebrospinal fluid, EM encephalomyelitis, I $g$ immunoglobulin, MOG myelin oligodendrocyte glycoprotein, MRZ measles, rubella and zoster virus, MS multiple sclerosis, NMDAR N-methyl-D-aspartate receptor, NMOSD neuromyelitis optica spectrum disorder, PPMS primary progressive MS, PML progressive multifocal leukoencephalopathy, PRES posterior reversible encephalopathy syndrome, SPMS secondary progressive MS, WCC white cell count

a Just one borderline MOG-IgG result found among 290 patients with PPMS $(n=174)$ or SPMS $(n=116)$ in a recent study [29]

${ }^{b}$ Measles $(M)$, rubella $(R)$, and zoster $(Z)$ reaction: Intrathecal synthesis against at least two of these three viral agents (i.e., against $M+R, M+Z, R+Z$, or $M+R+Z$ ); part of the polyspecific, intrathecal humoral immune reaction in MS; present in around 70\% of MS patients but not at all, or only very rarely, in MOG- or AQP4IgG-positive patients (MOG-EM: 0/11; NMO: 1/42; “ADEM": 1/26) [3, 70, 71]

'Except in patients who were previously positive at levels clearly above the cut-off, in which case low-titer results may reflect true (spontaneous or treatmentrelated) decline in antibody levels

${ }^{\mathrm{d}}$ May be valid in the rare instances in which co-existing serum autoantibodies hamper serum analysis but not CSF analysis (false-negative serum test) elf confirmed in a second assay and IPND criteria for NMOSD are met, co-existence of MOG-EM and AQP4-NMOSD must be assumed

${ }^{f}$ Note, however, that preliminary reports suggest occasional co-incidence of MOG-EM and NMDAR encephalitis [61]; in such patients teratoma needs to be excluded [60]

${ }^{9}$ Note that CSF findings in MOG-EM (as well as in AQP4-NMOSD) may mimic CNS infection with neutrophil pleocytosis, impaired blood-CSF barrier function, and a lack of CSF-restricted oligoclonal bands [3, 40,51]. White cell counts in MOG-EM ranged between 6 and 306 cells/ $\mu$ (median 33; quartile range 13-125) in a recent European study [2]; WCC $\geq 100$ cells/ $\mu$ l were present at least once in $9 / 32(28.1 \%)$ patients; neutrophil granulocytes were present at least once in $9 / 14$ (64.3\%) patients with pleocytosis and available data (median $22 \%$ of all white cells; range $3-69 \%$ )

${ }^{\mathrm{h}}$ May be true positive in the rare cases in which MOG-EM and unrelated peripheral neuropathy of other cause co-exist 
play a role. Finally, intervals sufficient to detect imminent attacks in time have not yet been defined. Based on experience from studies on AQP4-IgG-positive NMOSD, in which serum antibody levels rise only very shortly before an attack [32], very close testing intervals may be required, which would make monitoring both expensive and challenging from a practical point of view. Accordingly, no general recommendation for regular monitoring of MOG-IgG titers for relapse prediction or treatment monitoring can currently be made.

Of note, some patients have been reported in whom MOG-IgG disappeared over time [2, 33-35]. Interestingly, many of these patients had monophasic disease. By contrast, MOG-IgG was detectable at the last follow-up in all patients $(n=18)$ with a relapsing disease course and available follow-up samples (mean interval 33 months since first testing; maximum follow-up period 10 years) in a recent study [2]. Disappearance of MOG-IgG after the initial attack might thus have prognostic implications, and re-testing of MOG-IgG-positive patients 6-12 months after the first attack might therefore be useful. However, there are some limitations: Most of the reported monophasic patients were children or juveniles, and most had ADEM. Moreover, no long-term data were provided for most cases. This is important, since titers may fall below cut-off temporarily following treatment with steroids, plasma exchange, or immunosuppressants (or even spontaneously) and rise again at a later disease stage; accordingly, (transient) seroconversion has also been observed in a few patients with relapsing disease [2, 14, 33]. It would therefore be challenging to base long-term treatment decisions solely on whether MOG-IgG disappears or not after a first attack. If long-term treatment with immunosuppressants or oral steroids is abandoned by reason of conversion to seronegativity, close monitoring of the patient's MOGIgG serostatus is highly recommended to confirm seronegativity in the long-term course. Before making a diagnosis of "monophasic" MOG-EM and thus a decision against longterm treatment, one should also take into account that the interval between first and second attack in relapsing MOGEM varies considerably among patients, with the second clinical attack occurring only after an interval of several years in some cases [3].

\section{Diagnostic criteria for MOG-EM}

There is an unmet need for diagnostic criteria for MOGEM. However, no specific clinical or radiological findings (except for the general requirement of a demyelinating CNS lesion) have yet been identified that are present in all MOG-IgG-positive patients and which would thus represent a diagnostic sine qua non. A lack of Dawson's finger lesions and ovoid/round lesions on brain MRI have been proposed to be typical for MOG-EM, but this awaits confirmation in independent and larger cohorts
$[36,37]$. We propose that for the time being MOG-EM should be diagnosed in all patients who meet all of the following criteria:

1. Monophasic or relapsing acute ON, myelitis, brainstem encephalitis, or encephalitis, or any combination of these syndromes

2. MRI or electrophysiological (visual evoked potentials in patients with isolated $\mathrm{ON}$ ) findings compatible with CNS demyelination

3. Seropositivity for MOG-IgG as detected by means of a cell-based assay employing full-length human MOG as target antigen

In patients with conditions considered "red flags" as defined in Table 4 and in whom MOG-IgG has not yet been confirmed in a second (and third if necessary), methodologically different cell-based assay, a diagnosis of "possible MOG-EM" should be made, especially in the context of clinical studies and treatment trials.

\section{Limitations and caveats}

It is a limitation that all recommendations given here are necessarily based on expert consensus, owing to a lack of systematic and prospective studies. Moreover, as a general caveat, it should be stressed that before a diagnosis of MOG-EM is made, all available information, including clinical, radiological, electrophysiological, and laboratory data, need to be taken into account, and differential diagnoses, some of which are listed in Table 4, need to be excluded. Most of the information given in a previous consensus paper on differential diagnosis in MS [38] is also pertinent to MOG-EM. Finally, while the criteria proposed here can certainly help in identifying pediatric patients at high risk of being positive for MOG-IgG, they are primarily intended for use in adults and adolescents. Indications for MOG-IgG testing in children do not need to be as rigorous as in adults, since MOG-IgG is thought to be much more common in children with acquired demyelinating disease (up to $70 \%$ depending on age) than in their adult counterparts $(\leq 1 \%$ in Western countries; probably $\leq 5 \%$ in Japan and other Asian countries because of lower MS prevalence). In consequence, the risk of an unfavorable ratio of FP to TP results outlined above is lower in children. While ADEM is the predominant clinical association in young children, in older children with MOG antibodies there is a shift towards presentation with $\mathrm{ON}$, myelitis, and/or brainstem symptoms [11].

\section{Conclusion}

Here, we give for the first time indications for MOG-IgG testing and propose preliminary criteria for the diagnosis of MOG-EM. While we believe that our recommendations are highly timely considering the large 
numbers of patients currently being tested, we are well aware that they reflect current knowledge in an evolving field and may need to be adjusted when new clinical and paraclinical data emerge and novel and optimized assays become available.

\begin{abstract}
Abbreviations
ADEM: Acute disseminated encephalomyelitis; ADEM-ON: ADEM with recurrent ON; AQP4: Aquaporin-4; CNS: Central nervous system; CRION: Chronic relapsing inflammatory optic neuropathy; CSF: Cerebrospinal fluid; EM: Encephalomyelitis; Gd: Gadolinium; IA: Immunoadsorption; IgA: Immunoglobulin A; IgG: Immunoglobulin G; IgM: Immunoglobulin M; IVMP: Intravenous methylprednisolone; LETM: Longitudinally extensive transverse myelitis; MOG: Myelin oligodendrocyte glycoprotein; MRI: Magnetic resonance imaging; MRZ: Measles, rubella and zoster virus; MS: Multiple sclerosis; NMO: Neuromyelitis optica; NMOSD: NMO spectrum disorder; OCB: Oligoclonal IgG bands; ON: Optic neuritis; PEX: Plasma exchange; PML: Progressive multifocal leukoencephalopathy; PPMS: Primary progressive MS; PRES: Posterior reversible encephalopathy syndrome; RRMS: Relapsing-remitting MS; SPMS: Secondary progressive MS; VEP: Visual evoked potentials; VS: Vertebral segments; WCC: White cell count
\end{abstract}

\section{Acknowledgements}

BW is grateful to the Dietmar Hopp Stiftung and to Merck Serono for funding research on MOG-lgG. AS is supported by La Marató de TV3 (20141830). We acknowledge financial support by Deutsche Forschungsgemeinschaft within the funding programme Open Access Publishing, by the Baden-Württemberg Ministry of Science, Research and the Arts and by Ruprecht-Karls-Universität Heidelberg.

\section{Funding}

The work of BW was supported by the Dietmar Hopp Foundation; Merck Serono; German Federal Ministry of Education and Research (Competence Network Multiple Sclerosis); Deutsche Forschungsgemeinschaft (funding programme Open Access Publishing); Baden-Württemberg Ministry of Science, Research and the Arts; and Ruprecht-Karls-Universität Heidelberg. The funding sources had no role in study conception or design, data collection, analysis, or interpretation, or any other aspect pertinent to the article. None of the authors has been paid to write this article by a pharmaceutical company or other agency. The corresponding authors have final responsibility for the decision to submit for publication.

\section{Availability of data and materials}

The datasets generated and/or analyzed during the current study are not publicly available but are available from the corresponding author upon reasonable request.

\section{Authors' contributions}

SJ and BW conceived the project. SJ collected and analyzed the data and wrote the first draft. All authors were involved in critically revising the manuscript for important intellectual content. All authors read and approved the final manuscript.

\section{Ethics approval and consent to participate}

Not applicable.

\section{Competing interests}

OA has received grants by the German Research Foundation (DFG), the German Ministry for Education and Research (BMBF) (KKNMS; for NEMOS NationNMO FKZ 01GI1602), speaking fees and travel grants by Bayer, Biogen, Genzyme, Medimmune, Merck, Novartis, Roche, Sanofi, and Teva. DF received one honorarium for a presentation from Biogen not related to the present paper.

KF serves on the advisory boards for Bayer Schering Pharma, Biogen Idec, Mitsubishi Tanabe Pharma Corporation, Novartis Pharma, Chugai Pharmaceutical, Ono Pharmaceutical, Nihon Pharmaceutical, Alexion Pharmaceuticals, and Medimmune; has received travel funding and speaker honoraria from Bayer Schering Pharma, Biogen Idec, Eisai Inc., Mitsubishi Tanabe Pharma Corporation, Novartis Pharma, Astellas Pharma Inc., Takeda Pharmaceutical Company Limited, Asahi Kasei Medical Co., Daiichi Sankyo, and Nihon Pharmaceutical; is on the editorial board for Clinical and Experimental Neuroimmunology; is an advisory board member for Sri Lanka Journal of Neurology; and received research support from Bayer Schering Pharma, Biogen Idec Japan, Asahi Kasei Medical, The Chemo-Sero-Therapeutic Research Institute, Teva Pharmaceutical, Mitsubishi Tanabe Pharma, Teijin Pharma, Chugai Pharmaceutical, OnoPharmaceutical, Nihon Pharmaceutical, Genzyme Japan, Ministry of Education, Science and Technology of Japan, and Ministry of Health, Welfare and Labor of Japan.

AJ is supported by the NHS National Specialised Commissioning Group for NMO and has been a consultant for Shire, Alexion, Terumo-BCT and Chugai pharmaceuticals and has received research funding from Biogen and Alexion pharamaceuticals.

HJK has lectured, consulted, and received honoraria from Bayer Schering Pharma, Biogen, Genzyme, HanAll BioPharma, Medlmmune, Merck Serono, Novartis, Teva-Handok, and UCB; received a grant from the Ministry of Science, ICT \& Future Planning; and accepted research funding from Genzyme, Kael-GemVax, Merck Serono, Teva-Handok, and UCB; serves on a steering committee for Medlmmune; is a co-editor for the Multiple Sclerosis Journal - Experimental, Translational, and Clinical, and an associated editor for the Journal of Clinical Neurology.

IK has received honoraria for consultancy or lectures and travel reimbursement from Bayer Health Care, Biogen Idec, Chugai, Novartis, Shire and Roche and grant support from Biogen Idec, Novartis, Chugai and Diamed. TK has received travel expenses and personal compensations from Bayer Healthcare, Teva Pharma, Merck-Serono, Novartis, Sanofi Genzyme and Biogen-ldec as well as grant support from Chugai Pharma and Novartis. ML receives support from Quest Diagnostics.

JP has received support for scientific meetings and honorariums for advisory work from Merck Serono, ABIDE, Biogen Idec, Novartis, Alexion, Medimmune, Teva, Chugai Pharma and Bayer Schering, and unrestricted grants from Merck Serono, Novartis, Biogen Idec and Bayer Schering. Grants from the MS society. GMSI, NIHR and Guthy- Jackson Foundation for research studies. She runs a nationally commissioned service for neuromyelitis optica and congenital myasthenia. FP has received honoraria and research support from Alexion, Bayer, Biogen, Chugai, MerckSerono, Novartis, Genyzme, Medlmmune, Shire, Teva, and serves on scientific advisory boards for Alexion, Medlmmune and Novartis. He has received funding from Deutsche Forschungsgemeinschaft (DFG Exc 257), German Federal Ministry for Education and Research (Competence Network Multiple Sclerosis), Guthy Jackson Charitable Foundation, EU Framework Program 7, National Multiple Sclerosis Society of the USA. KR has received research support from the German Federal Ministry of Education and Research (BMBF/KKNMS, Competence Network Multiple Sclerosis) and Novartis as well as travel grants or speaking fees from the Guthy Jackson Charitable Foundation, Bayer Healthcare, Biogen Idec, Merck Serono, sanofi-aventis/Genzyme, Teva Pharmaceuticals, and Novartis. AS is supported by La Marató de TV3 (20141830).

$\mathrm{CT}$ has received honoraria for consultation and expert testimony from Bayer Vital $\mathrm{GmbH}$, Biogen Idec/GmbH, Genzyme $\mathrm{GmbH}$ and Novartis Pharmaceuticals. None of this interfered with the current report. BGW receives royalties from RSR Ltd., Oxford University, Hospices Civil de Lyon, and MVZ Labor PD Dr. Volkmann und Kollegen GbR for a patent of NMO-lgG as a diagnostic test for NMO and related disorders. He receives personal compensation for serving as a member of an adjudication committee for clinical trials in NMO being conducted by Medlmmune and Alexion pharmaceutical companies. He is a consultant for Caladrius Biosciences regarding a clinical trial for NMO. He receives personal compensation for serving on a data safety monitoring board for Novartis for clinical trials in MS.

The work of BW was supported by research grants from the Dietmar Hopp Foundation, from Merck Serono and from the German Federal Ministry of Education and Research (Competence Network Multiple Sclerosis).

SJ, NA, RCD, and JDS declare that they have no competing interests.

\section{Publisher's Note}

Springer Nature remains neutral with regard to jurisdictional claims in published maps and institutional affiliations.

\section{Author details}

${ }^{1}$ Molecular Neuroimmunology Group, Department of Neurology, University Hospital Heidelberg, Im Neuenheimer Feld 350, 69120 Heidelberg, Germany. ${ }^{2}$ NeuroCure Clinical Research Center, Charité - Universitätsmedizin Berlin, corporate member of Freie Universität Berlin, Humboldt-Universität zu Berlin, 
and Berlin Institute of Health, Berlin, Germany. ${ }^{3}$ Experimental and Clinical Research Center, Max Delbrueck Center for Molecular Medicine and Charité Universitätsmedizin Berlin, Berlin, Germany. ${ }^{4}$ Department of Neurology, Charité - Universitätsmedizin Berlin, Berlin, Germany. ${ }^{5}$ Department of Neurology, University of Düsseldorf, Düsseldorf, Germany. ${ }^{6}$ Department of Neurology, University of Southern Denmark, Odense, Denmark. ${ }^{7}$ Children's Hospital at Westmead, University of Sydney, Sydney, Australia. ${ }^{8}$ Department of Neurology, Hôpital de Hautepierre, Strasbourg Cedex, France. ${ }^{9}$ IRCCS, National Neurological Institute C. Mondino, Pavia, Italy. ${ }^{10}$ Department of Multiple Sclerosis Therapeutics, Tohoku University Graduate School of Medicine, Sendai, Japan. ${ }^{11} T$ The Walton Centre, Walton Centre NHS Foundation Trust, Liverpool, UK. ${ }^{12}$ Department of Neurology, Research Institute and Hospital of National Cancer Center, Goyang, South Korea. ${ }^{13}$ Department of Neurology, Ruhr University Bochum, Bochum, Germany. ${ }^{14}$ Institute of Clinical Neuroimmunology, Ludwig Maximilian University, Munich, Germany. ${ }^{15}$ Department of Neurology, Johns Hopkins Hospital, Cleveland, USA. ${ }^{16}$ Nuffield Department of Clinical Neurosciences, John Radcliffe Hospital, Oxford, UK. ${ }^{17}$ Service of Neurology, Hospital Clinic, and Institut d'Investigacions Biomèdiques August Pi i Sunyer (IDIBAPS), Universitat de Barcelona, Barcelona, Spain. ${ }^{18}$ Department of Neurology, Hannover Medical School, Hanover, Germany. ${ }^{19}$ Department of Neurology, Mayo Clinic, Rochester, MN, USA.

Received: 16 January 2018 Accepted: 2 April 2018 Published online: 03 May 2018

\section{References}

1. Mader S, Gredler V, Schanda K, Rostasy K, Dujmovic I, Pfaller K, Lutterotti A Jarius S, Di Pauli F, Kuenz B, et al. Complement activating antibodies to myelin oligodendrocyte glycoprotein in neuromyelitis optica and related disorders. J Neuroinflammation. 2011;8:184.

2. Jarius S, Ruprecht K, Kleiter I, Borisow N, Asgari N, Pitarokoili K, Pache F, Stich O, Beume LA, Hummert MW, et al. MOG-lgG in NMO and related disorders: a multicenter study of 50 patients. Part 1: frequency, syndrome specificity, influence of disease activity, long-term course, association with AQP4-lgG, and origin. J Neuroinflammation. 2016;13:279.

3. Jarius S, Ruprecht K, Kleiter I, Borisow N, Asgari N, Pitarokoili K, Pache F, Stich O, Beume LA, Hummert MW, et al. MOG-IgG in NMO and related disorders: a multicenter study of 50 patients. Part 2: epidemiology, clinical presentation, radiological and laboratory features, treatment responses, and long-term outcome. J Neuroinflammation. 2016;13:280.

4. Jarius S, Kleiter I, Ruprecht K, Asgari N, Pitarokoili K, Borisow N, Hummert MW, Trebst C, Pache F, Winkelmann A, et al. MOG-lgG in NMO and related disorders: a multicenter study of 50 patients. Part 3: brainstem involvement frequency, presentation and outcome. J Neuroinflammation. 2016;13:281.

5. Pache F, Zimmermann H, Mikolajczak J, Schumacher S, Lacheta A, Oertel FC, Bellmann-Strobl J, Jarius S, Wildemann B, Reindl M, et al. MOG-lgG in NMO and related disorders: a multicenter study of 50 patients. Part 4: afferent visual system damage after optic neuritis in MOG-IgG-seropositive versus AQP4-lgG-seropositive patients. J Neuroinflammation. 2016;13:282.

6. Sepulveda M, Armangue T, Martinez-Hernandez E, Arrambide G, Sola-Valls N, Sabater L, Tellez N, Midaglia L, Arino H, Peschl P, et al. Clinical spectrum associated with MOG autoimmunity in adults: significance of sharing rodent MOG epitopes. J Neurol. 2016;263:1349-60.

7. Kitley J, Waters P, Woodhall M, Leite MI, Murchison A, George J, Kuker W, Chandratre S, Vincent A, Palace J. Neuromyelitis optica spectrum disorders with aquaporin-4 and myelin-oligodendrocyte glycoprotein antibodies: a comparative study. JAMA Neurol. 2014;71:276-83.

8. Sato DK, Callegaro D, Lana-Peixoto MA, Waters PJ, de Haidar Jorge FM, Takahashi T, Nakashima I, Apostolos-Pereira SL, Talim N, Simm RF, et al. Distinction between MOG antibody-positive and AQP4 antibody-positive NMO spectrum disorders. Neurology. 2014;82:474-81.

9. Kitley J, Woodhall M, Waters P, Leite MI, Devenney E, Craig J, Palace J, Vincent A. Myelin-oligodendrocyte glycoprotein antibodies in adults with a neuromyelitis optica phenotype. Neurology. 2012;79:1273-7.

10. Ramanathan S, Dale RC, Brilot F. Anti-MOG antibody: the history, clinical phenotype, and pathogenicity of a serum biomarker for demyelination. Autoimmun Rev. 2016:15:307-24.

11. Reindl M, Jarius S, Rostasy K, Berger T: Myelin oligodendrocyte glycoprotein antibodies: How clinically useful are they? Curr Opin Neurol. 2017;30:295-301.
12. Zamvil SS, Slavin AJ. Does MOG Ig-positive AQP4-seronegative opticospinal inflammatory disease justify a diagnosis of NMO spectrum disorder? Neurol Neuroimmunol Neuroinflamm. 2015;2:e62.

13. Hohlfeld R, Dornmair K, Meinl E, Wekerle $H$. The search for the target antigens of multiple sclerosis, part 2: CD8+ T cells, B cells, and antibodies in the focus of reverse-translational research. Lancet Neurol. 2016;15:317-31.

14. Spadaro M, Gerdes LA, Krumbholz M, Ertl-Wagner B, Thaler FS, Schuh E, Metz I, Blaschek A, Dick A, Bruck W, et al. Autoantibodies to MOG in a distinct subgroup of adult multiple sclerosis. Neurol Neuroimmunol Neuroinflamm. 2016;3:e257.

15. Miyazaki T, Nakajima H, Motomura M, Tanaka K, Maeda Y, Shiraishi H, Tsujino A. A case of recurrent optic neuritis associated with cerebral and spinal cord lesions and autoantibodies against myelin oligodendrocyte glycoprotein relapsed after fingolimod therapy. Rinsho Shinkeigaku. 2016;56:265-9.

16. Tsuburaya RS, Miki N, Tanaka K, Kageyama T, Irahara K, Mukaida S, Shiraishi K, Tanaka M. Anti-myelin oligodendrocyte glycoprotein (MOG) antibodies in a Japanese boy with recurrent optic neuritis. Brain and Development. 2015;37:145-8.

17. Wildemann B, Jarius S, Schwarz A, Diem R, Viehöver A, Hähnel S, Reindl M, Korporal-Kuhnke M. Failure of alemtuzumab therapy to control MOG encephalomyelitis. Neurology. 2017;89:207-9.

18. Chalmoukou K, Alexopoulos H, Akrivou S, Stathopoulos P, Reindl M, Dalakas MC. Anti-MOG antibodies are frequently associated with steroid-sensitive recurrent optic neuritis. Neurol Neuroimmunol Neuroinflamm. 2015;2:e131.

19. Mayer MC, Breithaupt C, Reindl M, Schanda K, Rostasy K, Berger T, Dale RC, Brilot F, Olsson $T$, Jenne $D$, et al. Distinction and temporal stability of conformational epitopes on myelin oligodendrocyte glycoprotein recognized by patients with different inflammatory central nervous system diseases. J Immunol. 2013;191:3594-604.

20. Ramanathan S, Reddel SW, Henderson A, Parratt JD, Barnett M, Gatt PN, Merheb V, Kumaran RY, Pathmanandavel K, Sinmaz N, et al. Antibodies to myelin oligodendrocyte glycoprotein in bilateral and recurrent optic neuritis. Neurol Neuroimmunol Neuroinflamm. 2014;1:e40.

21. Matsuda R, Kezuka T, Umazume A, Okunuki Y, Goto H, Tanaka K. Clinical profile of anti-myelin oligodendrocyte glycoprotein antibody seropositive cases of optic neuritis. Neuroophthalmology. 2015;39:213-9.

22. Nishikawa Y, Oku H, Tonari K, Sugasawa J, Ikeda T. A case of CRION with high titer of MOG antibody. Neuro Ophthalmol Japan. 2016;33:27-31.

23. Oshiro A, Nakamura S, Tamashiro K, Fujihara K. Anti-MOG + neuromyelitis optica spectrum disorders treated with plasmapheresis. No To Hattatsu. 2016:48:199-203.

24. Spadaro M, Gerdes LA, Mayer MC, Ertl-Wagner B, Laurent S, Krumbholz M, Breithaupt C, Hogen T, Straube A, Giese A, et al. Histopathology and clinical course of MOG-antibody-associated encephalomyelitis. Ann Clin Transl Neurol. 2015;2:295-301.

25. Hacohen $Y$, Wong $Y Y$, Lechner $C$, Jurynczyk M, Wright S, Konuskan B, Kalser J, Poulat AL, Maurey H, Ganelin-Cohen E, et al. Disease Course and Treatment Responses in Children With Relapsing Myelin Oligodendrocyte Glycoprotein Antibody-Associated Disease. JAMA Neurol. 2018;epub ahead of print (https://doi.org/10.1001/jamaneurol.2017.4601).

26. Montcuquet A, Collongues N, Papeix C, Zephir H, Audoin B, Laplaud D, Bourre B, Brochet B, Camdessanche JP, Labauge P, et al. Effectiveness of mycophenolate mofetil as first-line therapy in AQP4-lgG, MOG-lgG, and seronegative neuromyelitis optica spectrum disorders. Mult Scler. 2017:23:1377-84.

27. Kister I, Paul F. Pushing the boundaries of neuromyelitis optica: does antibody make the disease? Neurology. 2015;85:118-9.

28. Jarius S, Wildemann B. Aquaporin-4 antibodies (NMO-lgG) as a serological marker of neuromyelitis optica: a critical review of the literature. Brain Pathol. 2013;23:661-83

29. Jarius S, Ruprecht K, Stellmann JP, Huss A, Ayzenberg I, Willing A, Trebst C, Pawlitzki M, Abdelhak A, Grüter T, et al: MOG-lgG in primary and secondary chronic progressive multiple sclerosis: a multicenter study of 200 patients and review of the literature. J Neuroinflammation 2018;15:88.

30. Wingerchuk DM, Banwell B, Bennett JL, Cabre P, Carroll W, Chitnis T, de Seze J, Fujihara K, Greenberg B, Jacob A, et al. International consensus diagnostic criteria for neuromyelitis optica spectrum disorders. Neurology. 2015;85:177-89.

31. Wingerchuk DM, Lennon VA, Pittock SJ, Lucchinetti CF, Weinshenker BG. Revised diagnostic criteria for neuromyelitis optica. Neurology. 2006;66:1485-9.

32. Jarius S, Aboul-Enein F, Waters P, Kuenz B, Hauser A, Berger T, Lang W, Reindl M, Vincent A, Kristoferitsch W. Antibody to aquaporin-4 in the longterm course of neuromyelitis optica. Brain. 2008;131:3072-80.

33. Probstel AK, Dornmair K, Bittner R, Sperl P, Jenne D, Magalhaes S, Villalobos A, Breithaupt $C$, Weissert $R$, Jacob $U$, et al. Antibodies to MOG are transient in childhood acute disseminated encephalomyelitis. Neurology. 2011;77:580-8. 
34. Di Pauli F, Mader S, Rostasy K, Schanda K, Bajer-Kornek B, Ehling R, Deisenhammer F, Reindl M, Berger T. Temporal dynamics of anti-MOG antibodies in CNS demyelinating diseases. Clin Immunol. 2011;138:247-54.

35. Hoftberger R, Sepulveda M, Armangue T, Blanco Y, Rostasy K, Cobo Calvo A, Olascoaga J, Ramio-Torrenta L, Reindl M, Benito-Leon J, et al. Antibodies to MOG and AQP4 in adults with neuromyelitis optica and suspected limited forms of the disease. Mult Scler. 2015;21:866-74.

36. Jurynczyk M, Geraldes R, Probert F, Woodhall MR, Waters P, Tackley G, Deluca G, Chandratre S, Leite MI, Vincent A, Palace J: Distinct brain imaging characteristics of autoantibody-mediated CNS conditions and multiple sclerosis. Brain. 2017;140:617-27.

37. Jurynczyk M, Tackley G, Kong Y, Geraldes R, Matthews L, Woodhall M, Waters $\mathrm{P}$, Kuker W, Craner M, Weir A, et al. Brain lesion distribution criteria distinguish MS from AQP4-antibody NMOSD and MOG-antibody disease. J Neurol Neurosurg Psychiatry. 2017;88:132-6.

38. Miller DH, Weinshenker BG, Filippi M, Banwell BL, Cohen JA, Freedman MS, Galetta SL, Hutchinson M, Johnson RT, Kappos L, et al. Differential diagnosis of suspected multiple sclerosis: a consensus approach. Mult Scler. 2008;14:1157-74.

39. Mariotto S, Ferrari S, Monaco S, Benedetti MD, Schanda K, Alberti D, Farinazzo A, Capra R, Mancinelli C, De Rossi N, et al: Clinical spectrum and IgG subclass analysis of anti-myelin oligodendrocyte glycoprotein antibodyassociated syndromes: a multicenter study. J Neurol 2017, 264:2420-2430.

40. Jarius S, Ruprecht K, Wildemann B, Kuempfel T, Ringelstein M, Geis C, Kleiter I, Kleinschnitz C, Berthele A, Brettschneider J, et al. Contrasting disease patterns in seropositive and seronegative neuromyelitis optica: a multicentre study of 175 patients. J Neuroinflammation. 2012;9:14

41. Baumann M, Grams A, Djurdjevic T, Wendel EM, Lechner C, Behring B, Blaschek A, Diepold K, Eisenkolbl A, Fluss J, et al: MRI of the first event in pediatric acquired demyelinating syndromes with antibodies to myelin oligodendrocyte glycoprotein. J Neurol. 2018;265:845-55.

42. van Pelt ED, Wong YY, Ketelslegers IA, Hamann D, Hintzen RQ. Neuromyelitis optica spectrum disorders: comparison of clinical and magnetic resonance imaging characteristics of AQP4-IgG versus MOG-lgG seropositive cases in the Netherlands. Eur J Neurol. 2016;23:580-7.

43. Ramanathan S, Prelog K, Barnes EH, Tantsis EM, Reddel SW, Henderson AP, Vucic S, Gorman MP, Benson LA, Alper G, et al. Radiological differentiation of optic neuritis with myelin oligodendrocyte glycoprotein antibodies, aquaporin-4 antibodies, and multiple sclerosis. Mult Scler. 2016;22:470-482.

44. Mealy MA, Whetstone A, Orman G, Izbudak I, Calabresi PA, Levy M. Longitudinally extensive optic neuritis as an MRI biomarker distinguishes neuromyelitis optica from multiple sclerosis. J Neurol Sci. 2015;355:59-63.

45. Akaishi T, Nakashima I, Takeshita T, Mugikura S, Sato DK, Takahashi T, Nishiyama S, Kurosawa K, Misu T, Nakazawa T, et al. Lesion length of optic neuritis impacts visual prognosis in neuromyelitis optica. J Neuroimmunol. 2016;293:28-33.

46. Storoni M, Davagnanam I, Radon M, Siddiqui A, Plant GT. Distinguishing optic neuritis in neuromyelitis optica spectrum disease from multiple sclerosis: a novel magnetic resonance imaging scoring system. J Neuroophthalmol. 2013;33:123-127.

47. Bouzar M, Daoudi S, Hattab S, Bouzar AA, Deiva K, Wildemann B, Reindl M, Jarius S. Neuromyelitis optica spectrum disorders with antibodies to myelin oligodendrocyte glycoprotein or aquaporin-4: Clinical and paraclinical characteristics in Algerian patients. J Neurol Sci. 2017;381:240-244.

48. Zhou L, Huang Y, Li H, Fan J, Zhangbao J, Yu H, Li Y, Lu J, Zhao C, Lu C, et al. MOG-antibody associated demyelinating disease of the CNS: a clinical and pathological study in Chinese Han patients. J Neuroimmunol. 2017;305:19-28.

49. Kim SM, Woodhall MR, Kim JS, Kim SJ, Park KS, Vincent A, Lee KW, Waters P. Antibodies to MOG in adults with inflammatory demyelinating disease of the CNS. Neurol Neuroimmunol Neuroinflamm. 2015;2:e163.

50. Matthews L, Marasco R, Jenkinson M, Kuker W, Luppe S, Leite Ml, Giorgio A, De Stefano N, Robertson N, Johansen-Berg H, et al. Distinction of seropositive NMO spectrum disorder and MS brain lesion distribution. Neurology. 2013;80:1330-7.

51. Jarius S, Paul F, Franciotta D, Ruprecht K, Ringelstein M, Bergamaschi R, Rommer P, Kleiter I, Stich O, Reuss R, et al. Cerebrospinal fluid findings in aquaporin-4 antibody positive neuromyelitis optica: results from 211 lumbar punctures. J Neurol Sci. 2011;306:82-90.

52. Reiber H. Cerebrospinal fluid-physiology, analysis and interpretation of protein patterns for diagnosis of neurological diseases. Mult Scler. 1998;4:99-107.

53. Reiber H, Teut M, Pohl D, Rostasy KM, Hanefeld F. Paediatric and adult multiple sclerosis: age-related differences and time course of the neuroimmunological response in cerebrospinal fluid. Mult Scler. 2009;15:1466-80.
54. Jurynczyk M, Messina S, Woodhall MR, Raza N, Everett R, Roca-Fernandez A, Tackley G, Hamid S, Sheard A, Reynolds G, et al. Clinical presentation and prognosis in MOG-antibody disease: a UK study. Brain. 2017;140:3128-38.

55. Frau J, Villar LM, Sardu C, Secci MA, Schirru L, Ferraro D, Coghe G, Lorefice L, Fenu $G$, Bedin R, et al. Intrathecal oligoclonal bands synthesis in multiple sclerosis: is it always a prognostic factor? J Neurol. 2018;265:424-30.

56. Andersson M, Alvarez-Cermeno J, Bernardi G, Cogato I, Fredman P, Frederiksen J, Fredrikson S, Gallo P, Grimaldi LM, Gronning M, et al. Cerebrospinal fluid in the diagnosis of multiple sclerosis: a consensus report. J Neurol Neurosurg Psychiatry. 1994;57:897-902.

57. Jarius S, Metz I, Konig FB, Ruprecht K, Reindl M, Paul F, Bruck W, Wildemann B. Screening for MOG-lgG and 27 other anti-glial and anti-neuronal autoantibodies in 'pattern II multiple sclerosis' and brain biopsy findings in a MOG-lgG-positive case. Mult Scler. 2016;22:1541-9.

58. Konig FB, Wildemann B, Nessler S, Zhou D, Hemmer B, Metz I, Hartung HP, Kieseier BC, Bruck W. Persistence of immunopathological and radiological traits in multiple sclerosis. Arch Neurol. 2008;65:1527-32.

59. Cobo-Calvo Á, Ruiz A, D'Indy H, Poulat AL, Carneiro M, Philippe N, DurandDubief F, Deiva K, Vukusic S, Desportes V, Marignier R. MOG antibodyrelated disorders: common features and uncommon presentations. J Neurol. 2017;264:1945-55

60. Titulaer MJ, Hoftberger R, lizuka T, Leypoldt F, McCracken L, Cellucci T, Benson LA, Shu H, Irioka T, Hirano M, et al. Overlapping demyelinating syndromes and anti-N-methyl-D-aspartate receptor encephalitis. Ann Neurol. 2014;75:411-28.

61. Fan S, Xu Y, Ren H, Guan H, Feng F, Gao X, Ding D, Fang F, Shan G, Guan T, et al. Comparison of myelin oligodendrocyte glycoprotein (MOG)-antibody disease and AQP4-IgG-positive neuromyelitis optica spectrum disorder (NMOSD) when they co-exist with anti-NMDA (N-methyl-D-aspartate) receptor encephalitis. Mult Scler Relat Disord. 2018;20:144-52.

62. Petzold A, Plant GT. Chronic relapsing inflammatory optic neuropathy: a systematic review of 122 cases reported. J Neurol. 2014;261:17-26.

63. Waters $P$, Woodhall $M, O^{\prime}$ Connor KC, Reindl M, Lang B, Sato DK, Jurynczyk M, Tackley G, Rocha J, Takahashi T, et al. MOG cell-based assay detects non-MS patients with inflammatory neurologic disease. Neurol Neuroimmunol Neuroinflamm. 2015;2:e89.

64. Peschl P, Schanda K, Zeka B, Given K, Bohm D, Ruprecht K, Saiz A, Lutterotti A, Rostasy K, Hoftberger R, et al. Human antibodies against the myelin oligodendrocyte glycoprotein can cause complement-dependent demyelination. J Neuroinflammation. 2017;14:208.

65. Jarius S, Franciotta D, Bergamaschi R, Wildemann B, Wandinger KP: IgM antibodies to aquaporin-4 in neuromyelitis optica and related disorders. Clin Chem Lab Med 2010; in press.

66. Jarius S, Eichhorn P, Albert MH, Wagenpfeil S, Wick M, Belohradsky BH, Hohlfeld R, Jenne DE, Voltz R. Intravenous immunoglobulins contain naturally occurring antibodies that mimic antineutrophil cytoplasmic antibodies and activate neutrophils in a TNFalpha-dependent and fcreceptor-independent way. Blood. 2007;109:4376-82.

67. Arnold DM, Crowther MA, Meyer RM, Carruthers J, Ditomasso J, Heddle NM, McLeod A, Kelton JG. Misleading hepatitis B test results due to intravenous immunoglobulin administration: implications for a clinical trial of rituximab in immune thrombocytopenia. Transfusion. 2010;50:2577-81.

68. Garcia L, Huh YO, Fischer HE, Lichtiger B. Positive immunohematologic and serologic test results due to high-dose intravenous immune globulin administration. Transfusion. 1987;27:503.

69. Cortese A, Franciotta D, Alfonsi E, Visigalli N, Zardini E, Diamanti L, Prunetti P, Osera C, Gastaldi M, Berzero G, et al. Combined central and peripheral demyelination: clinical features, diagnostic findings, and treatment. J Neurol Sci. 2016;363:182-7.

70. Jarius S, Eichhorn P, Franciotta D, Petereit HF, Akman-Demir G, Wick M, Wildemann $B$. The MRZ reaction as a highly specific marker of multiple sclerosis: re-evaluation and structured review of the literature. J Neurol. 2017;264:453-66.

71. Reiber H, Ungefehr S, Jacobi C. The intrathecal, polyspecific and oligoclonal immune response in multiple sclerosis. Mult Scler. 1998;4:111-7. 